\title{
A Randomized, Prospective Study to Compare the Efficacy and Safety of Omega-3 Fatty Acids as Additional Therapy to Methotrexate versus Methotrexate Monotherapy in the Management of Moderate to Severe Plaque Psoriasis
}

\author{
Ankita Bedwal, Kavitha Rajarathna, T.N. Revathi
}

Department of Pharmacology, Bangalore Medical College \& Research Institute, Bangalore, India Department of Dermatology, Bangalore Medical College \& Research Institute, Bangalore, India

\begin{abstract}
Psoriasis is a chronic, inflammatory, hyperproliferative, immune-mediated skin disorder, having a prevalence of $0.44-2.8 \%$ in India. Methotrexate is a widely used systemic regimen for moderate to severe psoriasis. Supplementation of omega-3 fatty acids has decreased the severity of the disease in some studies. This study aimed to assess the efficacy and safety of omega-3 fatty acids as an add-on to methotrexate versus methotrexate monotherapy in patients with moderate to severe plaque psoriasis. A total of 40 patients with moderate to severe plaque psoriasis were recruited in the study and randomized into two groups of 20 patients each. Group $\mathrm{O}$ received omega-3 fatty acids as add-on to methotrexate and Group M received methotrexate monotherapy. Psoriasis Area and Severity Index (PASI), Dermatology Life Quality Index (DLQI), Physician's Global Assessment (PGA) were assessed at baseline, 4 weeks, 8 weeks and 12 weeks of treatment. The adverse events were assessed throughout the study period. There was a significant decrease in PASI and DLQI scores from baseline to end of 12 weeks in both groups $(p<0.001)$ but the difference between the two groups was not significant ( $p>0.05)$. A higher number of patients had a PGA score of 0 or 1 at the end of 12 weeks in Group O (60\%) as compared to Group M (40\%) but the difference between the groups was not significant ( $\mathrm{p}=0.34$ ). The treatment was well tolerated in both the groups, with most common adverse events being nausea, diarrhea and epigastric pain. This study showed that the treatment in both the groups was equally effective in decreasing the severity of psoriasis and was well tolerated.
\end{abstract}

Keywords : Plaque psoriasis, methotrexate, omega-3 fatty acids, Psoriasis Area and Severity Index

\section{Introduction}

Psoriasis is a common, chronic, disfiguring, inflammatory and proliferative condition of the skin, in which genetic and environmental influences have a critical role. It affects about $2 \%$ of the population and is characterized by pronounced hyperproliferation of keratinocytes, markedly increased vascularization of the skin, fibroblast activation and leucocyte infiltration. ${ }^{1}$ The most characteristic lesions seen are red, scaly, sharply demarcated, indurated plaques,

Corresponding author: Kavitha Rajarathna, Department of Pharmacology, Bangalore Medical College, Bangalore, India. Email: kavitharajarathna@gmail.comm

Received: 30 November 2020. Revised: 15 January 2021. Published: 1 April 2021 
present particularly over extensor surfaces and scalp. ${ }^{2}$

The choice of treatment in psoriasis is influenced by factors like the severity of the disease, the effectiveness of a given medication and its side effects, the patient's quality of life and the ease of treatment. ${ }^{3}$ The treatment options include topical and systemic therapies as well as phototherapy. The topical treatments include emollients, tar, corticosteroids and tacrolimus. The systemic treatment includes drugs like methotrexate, cyclosporin and acitrecin. ${ }^{4}$

Methotrexate, introduced in 1958, is an economical and effective antipsoriatic agent used for psoriasis. It acts by inhibiting DNA synthesis by competitive inhibition of the enzyme dihydrofolate reductase leading to anti-mitotic action on psoriatic skin. It was originally used in psoriasis due to its effect on rapidly dividing keratinocytes, however it is also known to have anti-inflammatory and immune-modulating properties. ${ }^{5}$ The anti-inflammatory action of methotrexate is attributed to increased adenosine concentration which is a result of intracellular 5 - a mino imidazole-4 - carbox a mide ribonucleotide (AICAR) accumulation. ${ }^{6}$

Omega-3 fatty acid supplementation has shown to decrease the severity of psoriasis in few studies. It acts by altering the epidermal and blood cell membrane lipid composition. ${ }^{7}$ In psoriatic lesions, there are profound changes in the metabolism of eicosanoids with increased concentrations of free arachidonic acid and its pro-inflammatory metabolites. ${ }^{8}$ The arachidonic acid and its metabolite, leukotriene 4 (LTB4), are considered to be the principal mediators of inflammation in psoriasis. When cyclooxygenase or lipoxygenase metabolizes eicosapentaenoic acid (EPA) instead of arachidonic acid in cell membranes, it leads to the formation of metabolites like leukotriene B5 (LTB5), which are less potent inflammatory mediators. Hence, replacement of arachidonic acid by alternative precursor omega-3 fatty acids (especially EPA and docosahexanoic acid, DHA), is of therapeutic advantage in psoriasis. ${ }^{5,79}$ Therefore, this study was conducted to evaluate the efficacy and safety of the addition of omega- 3 fatty acids to methotrexate in the treatment of psoriasis as previous studies have demonstrated its possible effect on evolution of the disease. The aim of the study was to assess the efficacy and safety of omega-3 fatty acids as add on therapy to methotrexate in moderate to severe psoriasis vulgaris.

\section{Methods}

This research was a prospective, comparative, parallel, open label study conducted on outpatients attending the Department of Dermatology of Victoria Hospital, Bowring Hospital and Lady Curzon Hospital attached to Bangalore Medical College and Research Institute, Bangalore, India from November 2015 to May 2017. The study was approved by Institutional Ethics Committee.

Taking the prevalence of psoriasis as $2.8 \%$, power of the study as $80 \%$ and confidence limit at $95 \%$, to detect significant difference in efficacy and safety between two groups, a minimum of 10 patients were required in each group. For a better validation of results and due to limited number of studies, we included 20 patients in each group. CONSORT guidelines are attached in the supplementary files.

\section{Inclusion Criteria}

Patients with moderate or severe plaque psoriasis (PASI $>10$ or PASI $\leq 10$ and DLQI > 10) between 18- 60 years who were willing to give written informed consent and 
had normal Liver Function Tests and Renal Function Tests.

Demographic data, medical history, concomitant medications, physical examination and clinical examination were recorded in the study proforma at baseline visit (Visit 1). Follow up visits were at the end of 4 weeks (visit 2), 8 weeks (visit 3) and 12 weeks (visit 4) after administering the study drugs. PASI, DLQI, and PGA were recorded at each visit.

\section{Exclusion Criteria}

Patients with:

1. Renal, hepatic or lung disease

2. Pregnancy or lactation

3. Anaemia (haemoglobin $<9 \mathrm{gm} / \mathrm{dL}$ ), leucopenia, or thrombocytopenia

4. Active infections, immunodeficiency states, concomitant skin disorders

5. Hypersensitivity to any of the study drugs, topical or systemic treatment for psoriasis, or methotrexate intake within 6 weeks of study initiation.

After obtaining approval and clearance from the Institutional Ethics Committee, a total of 40 patients were enrolled in the study. The study subjects fulfilling the inclusion criteria were randomly assigned into two groups of twenty patients using random number table. Group $\mathrm{O}$ received omega 3 fatty acids 300 mg (EPA $120 \mathrm{mg}+$ DHA $80 \mathrm{mg}$ ) once a day along with methotrexate $7.5 \mathrm{mg} /$ week. Group $\mathrm{M}$ received methotrexate monotherapy of 7.5 $\mathrm{mg} /$ week.

\section{Statistical Analysis}

The results obtained were analyzed using mean, standard deviation, repeated measures ANOVA, paired t- test and unpaired t-test for continuous variables. Chi- square test and Fisher's exact test were used for categorical variables.

\section{Results and Discussion}

A total of 40 patients were enrolled in the study based on the inclusion and exclusion criteria. The participant recruitment and treatment flow diagram are depicted in Figure 1 .

\section{Baseline Characteristics}

The baseline demographic, clinical characteristics and disease severity of the patients included in the study showed no significant difference between the two groups $(\mathrm{p}>0.05)$ as depicted in Table 1.

\section{Efficacy Measurement}

There was a significant decrease in PASI from baseline to end of 12 weeks in both the groups (Figure 2). The mean difference in PASI score from baseline to end of week 12 in Group M was $11.52 \pm 3.6(\mathrm{p}<0.001)$ whereas in Group $\mathrm{O}$, it was $11.3 \pm 3.9(\mathrm{p}<0.001)$, however there was no significant difference between the two groups $(p=0.09)$. An improvement of $50 \%$ in PASI score (PASI 50) was noted in $75 \%$ of the patients in Group $\mathrm{M}$ as compared to $95 \%$ of the patients in Group $\mathrm{O}$ at the end of 12 weeks of treatment but the difference was not statistically significant ( $p>0.05)$. However, a significant number of patients achieved PASI 50 in Group O (45\%) as compared to Group $M(15 \%)$ at the end of 8 weeks $(p=0.04)$.

PASI is a measure of overall psoriasis severity that assesses BSA, erythema, induration, and scaling. A $75 \%$ reduction in the PASI score (PASI 75) is the current benchmark of primary endpoints for most clinical trials of psoriasis. However, according to Carlin et al, PASI 50 (50\% reduction in PASI score) equates to a clinically meaningful improvement in psoriasis. $^{10,11}$

In a meta-analysis by West et al that included eleven studies, it was observed that $45.2 \%$ of patients achieved an improvement of $75 \%$ in 




Figure 1. Participants Recruitment and Treatment Flow Diagram

PASI score (PASI 75) at 12 or 16 weeks as the primary endpoint. ${ }^{12}$ However, in our study, only $5 \%$ of patients was found to achieve PASI 75. This may be attributed to the fact that our study duration was of 12 weeks and a fixed minimum dose of $7.5 \mathrm{mg} /$ week of methotrexate was administered for 12 weeks in contrast to the flexible dose adjustment seen in other studies where dose was adjusted based on the response of the patients. In the present study, it was observed that higher number of patients achieved PASI 50 at the end of 8 weeks in Group $\mathrm{O}$ as compared to Group $\mathrm{M}$, which suggests that the addition of omega 3 fatty acids to methotrexate can lead to an earlier improvement in the severity of the disease. However, this difference was overcome at the end of 12 weeks in between the treatment groups. The intake of omega 3 fatty acids, EPA and DHA leads to the formation of hydroxylated metabolites through 15-lipoxygenase, i.e., 15-hydroxyeicosapentaenoic acid, and 15-hydroxydocosahexaenoic acid. Both these substances are potent inhibitors of the 5-lipoxygenase of mononuclear cells, thus limiting the synthesis of proinflammatory leukotrienes LTB4, LTC4, and LTD4, limiting the spreading of the inflammatory process and hence reducing the symptoms of psoriasis. ${ }^{9}$ This additional anti-inflammatory action of omega 3 fatty acids might be responsible for the earlier improvement in disease severity. 
Table 1. Demographic Profile

\begin{tabular}{|c|c|c|c|c|}
\hline Variable & & $\begin{array}{c}\text { Group M } \\
\text { n }(\%)\end{array}$ & $\begin{array}{c}\text { Group O } \\
\text { n }(\%)\end{array}$ & \\
\hline \multirow{4}{*}{ Age (Years) } & $0-20$ & $0(0)$ & $1(5)$ & \multirow{4}{*}{$\mathrm{p}=0.06$} \\
\hline & $21-40$ & $4(20)$ & $10(500$ & \\
\hline & $41-60$ & $15(75)$ & $9(45)$ & \\
\hline & $>60$ & $1(5)$ & $0(0)$ & \\
\hline \multirow{2}{*}{ Gender } & Male & $13(65)$ & $10(50)$ & \multirow{2}{*}{$p=0.3$} \\
\hline & Female & $7(35)$ & $10(50)$ & \\
\hline \multirow{2}{*}{$\begin{array}{l}\text { Family History of } \\
\text { Psoriasis }\end{array}$} & Present & $4(20)$ & $2(10)$ & \multirow{2}{*}{$\mathrm{p}=0.6$} \\
\hline & Absent & $16(80)$ & $18(90)$ & \\
\hline \multirow[t]{2}{*}{ Smoker } & Yes & $14(70)$ & $9(45)$ & \multirow[t]{2}{*}{$p=0.1$} \\
\hline & No & $6(30)$ & $11(55)$ & \\
\hline \multirow[t]{2}{*}{ Alcoholic } & Yes & $10(50)$ & $7(35)$ & \multirow[t]{2}{*}{$p=0.3$} \\
\hline & No & $10(50)$ & $13(65)$ & \\
\hline PASI Score & Mean & $20.9 \pm 8.04$ & $18.5 \pm 5.5$ & $\mathrm{p}=0.16$ \\
\hline DLQI score & Mean & $17.7 \pm 5.03$ & $18.7 \pm 4.6$ & $\mathrm{p}=0.5$ \\
\hline \multirow{4}{*}{ PGA scores } & $0-2$ & $1(5)$ & $1(5)$ & \multirow{4}{*}{$\mathrm{p}=0.68$} \\
\hline & 3 & $10(50)$ & $11(55)$ & \\
\hline & 4 & $7(35)$ & $8(40)$ & \\
\hline & 5 & $2(10)$ & $0(0)$ & \\
\hline
\end{tabular}

$\mathrm{n}=20$

Psoriasis has a significant negative impact on patient's quality of life (QOL). DLQI, which is used for assessment of QOL, consists of 10 questions covering six domains, giving an overall range of $0-30$. Lower scores mean better quality of life. ${ }^{13}$

The mean reduction in DLQI score from baseline to end of week 12 in Group M was $12.7 \pm 3.5(\mathrm{p}<0.001)$ and in Group O was 13.15 $\pm 3.6(p<0.001)$ but the difference between the two groups was not statistically significant $(p=$ $0.6)$ as depicted in Figure 3. This indicates that methotrexate by itself, is highly effective in improving the quality of life of patients with psoriasis.
A higher number of patients had a PGA score of 0 or 1 at the end of 12 weeks in Group O $(60 \%)$ as compared to Group M (40\%) but the difference was not statistically significant $(p=0.34)$ (Figure 4). The PGA scores at each visit have been shown in Table 2.

PGA is another widely used system employed in psoriasis clinical trials for the assessment of psoriasis severity. ${ }^{14}$ In this study, it was observed that both the treatments were equally effective in decreasing the severity of psoriasis from baseline to end of 12 weeks as inferred from the decrease in PGA score. In a study by Reich et al, $22.1 \%$ of the patients in the methotrexate group achieved a score of 0 or 1 at the end of 12 weeks. ${ }^{15}$ In our study, almost 


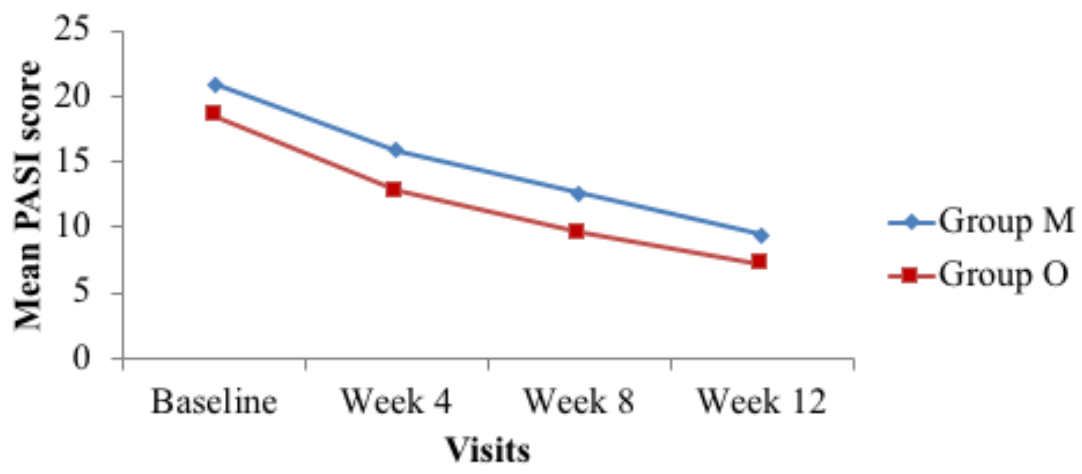

Figure 2. Mean PASI scores

$\mathrm{p}<0.001$ (within group), $\mathrm{p}=0.09$ (in between groups)

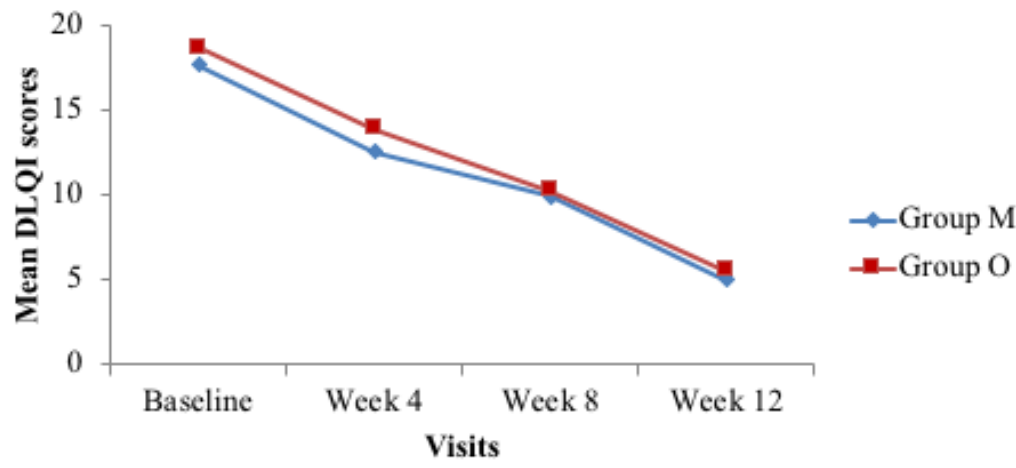

Figure 3. Mean DLQI scores

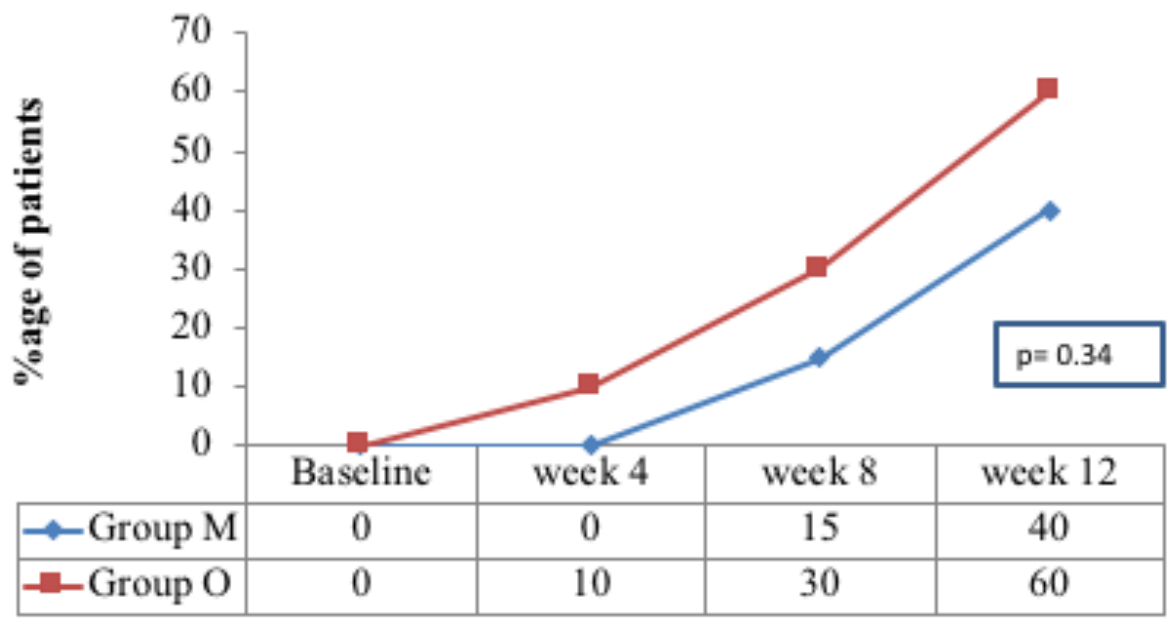

Figure 4. Patients with PGA score of 0 and 1

half of the patients in both the groups had mild or no skin lesions (score 0,1$)$ at the end of 12 weeks but a statistically significant difference between the two groups was not detected.

The treatment was well tolerated in both the groups. During the study duration, the most common treatment-emergent adverse events reported were nausea, diarrhea and epigastric pain (Table 3). However, there was no significant difference in the occurrence of adverse events between the two groups 
Table 2. PGA Scores

\begin{tabular}{ccccccc}
\hline \multirow{2}{*}{ PGA } & \multicolumn{2}{c}{ End of Week 4 } & \multicolumn{2}{c}{ End of Week 8 } & \multicolumn{2}{c}{ End of Week 12 } \\
\cline { 2 - 7 } & $\begin{array}{c}\text { Group M } \\
\mathrm{n}(\%)\end{array}$ & $\begin{array}{c}\text { Group O } \\
\mathrm{n}(\%)\end{array}$ & $\begin{array}{c}\text { Group M } \\
\mathrm{n}(\%)\end{array}$ & $\begin{array}{c}\text { Group O } \\
\mathrm{n}(\%)\end{array}$ & $\begin{array}{c}\text { Group M } \\
\mathrm{n}(\%)\end{array}$ & $\begin{array}{c}\text { Group O } \\
\mathrm{n}(\%)\end{array}$ \\
\hline $0-1$ & $0(0)$ & $2(10)$ & $3(15)$ & $6(30)$ & $8(40)$ & $12(60)$ \\
2 & $4(20)$ & $7(35)$ & $10(50)$ & $8(40)$ & $11(55)$ & $7(35)$ \\
3 & $14(70)$ & $10(50)$ & $6(30)$ & $6(30)$ & $1(5)$ & $1(5)$ \\
$4-5$ & $2(19)$ & $1(5)$ & $1(5)$ & $0(0)$ & $0(0)$ & $0(0)$ \\
\hline
\end{tabular}

Table 3. Adverse Events

\begin{tabular}{ccc}
\hline Adverse Events & $\begin{array}{c}\text { Group M } \\
\text { n (\%) }\end{array}$ & $\begin{array}{c}\text { Group O } \\
\text { n (\%) }\end{array}$ \\
\hline Nausea & $1(5)$ & $2(10)$ \\
Diarrhea & $1(5)$ & $0(0)$ \\
Epigastric Pain & $1(5)$ & $1(5)$ \\
Skin Irritation & $0(0)$ & $1(5)$ \\
Bloating & $1(5)$ & $0(0)$ \\
Fever & $1(5)$ & $0(0)$ \\
Total Patients & $5(25)$ & $4(20)$ \\
\hline
\end{tabular}

$p=1.0$ (between the two groups)

$(p=1.0)$. All the adverse events were mild to moderate in severity with no serious adverse events.

Methotrexate is a relatively safe drug in the dosage used in dermatology (7.5 $\mathrm{mg}$ to $25 \mathrm{mg}$ / week). ${ }^{16}$ Loss of appetite, nausea, diarrhoea and mouth ulcers are the most common side effects. ${ }^{17}$ Dose dependent adverse effects occur mainly in the cells that proliferate faster, thereby leading to a high incidence of gastrointestinal side effects. These side effects are usually temporary, but changes in dose, and/or supplemental folic acid tablets are required. ${ }^{16}$

With respect to the long-term safety of methotrexate, previous trials have shown the occurrence of hepatic adverse events, leading to discontinuation of the therapy but it was observed with a high dose of methotrexate $(15-25 \mathrm{mg} /$ week). These results suggest that initiating methotrexate at low dose and increasing it as indicated and as tolerated may be a safe strategy for long term use of this drug. ${ }^{3,15,18}$

The low incidence of side effects in the present study can be attributed to the use of lower fixed dose of methotrexate, regular evaluation of patients and the supplementation of $5 \mathrm{mg}$ of folic acid daily (except for Methotrexate days) in all the patients.

The study demonstrated that methotrexate, by itself, is effective in reducing the severity 
of disease in patients with moderate to severe psoriasis. The addition of omega- 3 fatty acids to methotrexate helped in achieving clinically meaningful improvement in severity in higher number of patients by the end of 8 weeks, however this difference was not observed at the end of 12 weeks.

To the best of our knowledge, this is the first study done to compare the efficacy and safety of omega 3 fatty acids as add on to methotrexate versus methotrexate monotherapy in mild to moderate psoriasis in Indian setup. The prospective, randomized study design and use of standard validated tools for assessing efficacy of treatments has added strength to the study.

The study was limited by its relatively short time frame, as 12 weeks is not sufficiently long enough to evaluate long term efficacy or safety of treatments in a chronic disease like psoriasis and dose titration of methotrexate was not done. The dose of Omega 3 fatty acids was also kept low. Smaller sample size and its open label nature were the other limiting factors. These might be the reasons that we were not able to detect a significant difference between the two treatment modalities. Another limitation is that the study was conducted about 4 years back. Therefore, further studies with larger sample size and longer study duration are required to evaluate the efficacy and cost effectiveness of omega 3 fatty acids in psoriasis.

\section{Conclusion}

A significant decline in the severity of psoriasis was observed in both the groups from baseline to end of 12 weeks but the difference between the two groups was not significant. The side effects observed were mild to moderate in nature. Hence, methotrexate monotherapy, by itself, is effective in reducing the severity of disease in patients with moderate to severe psoriasis. However, further studies with larger sample size and longer duration are warranted to detect the efficacy and ideal dose of omega-3 fatty acids supplementation in psoriasis.

\section{Acknowledgement}

The authors would like to thank the faculty and postgraduate students from the Department of Pharmacology and Department of Dermatology, Bangalore Medical College \& Research Institute, Bengaluru, for their support in conducting the project work.

\section{Funding}

None

\section{Conflict of Interest}

None declared

\section{References}

1. Burns T, Breathnach S, Cox N, Griffiths C. Psoriasis in Griffiths C, Barker J: Rook's Textbook of Dermatology, 8th ed. Wiley Blackwell. Vol 1 2010: 871-930.

2. Christophers E, Mrowietz U. Psoriasis in Freedberg IM, Eisen AZ, Wolff K, Austen KF, Goldsmith LA, Katz S: Fitzpatrick's Dermatology In General Medicine, 6th ed. McGraw-Hill Professional. Vol 1 2003: 476-494.

3. Heydendael VM, Spuls PI, Opmeer BC, de Borgie CA, Reitsma JB, Goldschmidt WF et al. Methotrexate versus cyclosporine in moderate-to-severe chronic plaque psoriasis. New England Journal of Medicine. 2003; 349(7):658-665.

4. Kuchekar AB, Pujari RR, Kuchekar SB, Dhole SN, Mule PM. Psoriasis: A comprehensive review. International Journal of Pharmaceutical and Life Sciences. 2011; 2(6):857-877.

5. Haider S, Wahid Z, Saher N, Riaz F. Efficacy of Methotrexate in patients with plaque type psoriasis. Pakistan Journal of Medical Sciences. 2014; 30(5):1050- 
1053.

6. Danielsen K, Olsen AO, Wilsgaard T, Furberg AS. Is the prevalence of psoriasis increasing? A 30-year follow-up ofa population-based cohort. British Journal of Dermatology. 2013; 168:1303-10.

7. Mari NL, Simao AN, Dichi I. n-3 polyunsaturated fatty acids supplementation in psoriasis: a review. Nutrire. 2017; 42:5.

8. Mayser P, Grimm H, Grimminger F. n-3 Fatty acids in psoriasis. British Journal of Nutrition. 2002; 87, Suppl. 1: S77-S82.

9. Márquez-Balbás G, Sánchez-Regaña $M$, Millet U. Study on the use of omega-3 fatty acids as a therapeutic supplement in treatment of psoriasis. Clinical Cosmetic and Investigational Dermatology. 2011; 4:73-77.

10. Dogra S, Mahajan R. Psoriasis: Epidemiology, clinical features, comorbidities, and clinical scoring. Indian Journal of Dermatology. 2016; 7(6):47180.

11. Carlin CS, Feldman SR, Krueger JG, Menter A, Krueger GG. A 50\% reduction in the Psoriasis Area and Severity Index (PASI 50) is a clinically significant endpoint in the assessment of psoriasis. Journal of the American Academy of Dermatology. 2004; 50:859-66.

12. West J, Ogston S, Foerster J. Safety and Efficacy of Methotrexate in Psoriasis: A Meta-analysis of Published Trials. PLoS One. 2016; 11(5): $\mathrm{e} 0153740$.

13. Feldman SR. Psoriasis assessment tools in clinical trials. Annals of the Rheumatic Diseases. 2005; 64(2):ii65-ii68.

14. Menter A, Gottlieb A, Feldman SR, Van Voorhees AS, Leonardi CL, Gordon KB, et al. Guidelines of care for the management of psoriasis and psoriatic arthritis. Journal of the American Academy of Dermatology. 2008; 58(5): 826-50.

15. Reich K, Langley RG, Papp KA, Ortonne
J-P, Unnebrink K, Kaul M, et al. A 52week trial comparing briakinumab with methotrexate in patients with psoriasis. New England Journal of Medicine. 2011; 365 (17):1586-1596.

16. Souza CFD, Suarez OMZ, Silva TFM, Gorenstein ACLA, Quintella LP, Avelleira JCR. Ulcerations due to methotrexate toxicity in a psoriasis patient. Anais Brasileiros de Dermatologia. 2016; 91(3):375-7.

17. Ngan V. DermNet New Zealand DermNet NZ. [cited 2017 Sep 22]; Available from: http://www.dermnetnz.org/topics/ guidelines-for-the-management-ofpsoriasis.

18. Kumari S. Methotrexate Induced Hepatotoxicity and its Management. International Journal of Pharmaceutical Sciences and Research. 2016; 5(9):14771481. 